\title{
Guillaume G.
}

Vossloh Signaling Department, Siema Applications, engineering department

\section{Huguet S.}

Vossloh Signaling Department, Siema Applications, engineering department

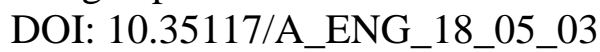

\section{Best practices for the monitoring of railway assets}

\begin{abstract}
Vossloh has acquired throughout the years a large experience in the monitoring of railway assets. Predictive maintenance applied to turnout monitoring improves the availability time of the asset. The main types of defects that can be monitored and the corresponding measurements are reviewed. High-level indicators help the maintenance team qualify the status of the turnout, and predictive maintenance can be used to anticipate some degradations. A data mining study is presented on a dataset from real turnouts.
\end{abstract}

Keywords: Railway, Monitoring; Turnout; Predictive; Maintenance; Sensors; Measurement

\section{Introduction}

Performance based contracts create an incentive for predictive maintenance. Subcontractors need dedicated tools, including remote monitoring systems for turnouts, to optimize maintenance costs and to commit about track availability. Vossloh has been providing monitoring systems to the French railway (SNCF) for more than 12 years, through its subsidiary Siema Applications. This document presents the best practices for the monitoring of the turnouts, based on Vossloh experience and its customer's feedbacks.

\section{Vossloh signaling department Monitored devices}

- Remote Event Monitoring

This monitoring system provides a large range of data acquisition analog and digital interfaces able to check in real time information gathered from signaling equipment along the track, or in any technical or signaling room. Energy station, communication devices, signaling panels, signaling equipment, track circuit, level crossing... are some examples of monitored assets.

- Remote Security Monitoring

This system allows real time detection of an electrical cable cut. The alarm is sent in less than 20 seconds to concerned people (police, security team, maintenance team).

- Remote Condition Monitoring

These products detect trends and tendency of the equipment behavior in order to anticipate failures and warn the maintenance operator that something has happened or will happen. One product is dedicated to monitor switches \& crossings while another one monitors track circuits. Both systems are developed on a common software kernel and can be easily merged for the user.

Both include an event recorder, a user configuration tool, a local diagnostic tool, and a web server diagnostic tool.

They acquire a wide range of measurements in real time, process them locally, then archive them at an offset station and perform analyses, which are either immediate or differed, in order to predict maintenance actions and anticipate breakdowns or interventions. 


\section{Main achievements}

Vossloh has acquired a large experience in monitoring of railway assets, coming from numerous contracts including:

- COSEA/INEO (Tours Bordeaux line, France)

- SNCF (French national railway Company)

- OCVIA (Nîmes Montpellier line, France)

- EIFFAGE (Le Mans Rennes line, France)

- Infrabel (Belgium railway company)

- ONCF (Morocco)

- $\quad$ SNCFT (Tunisia)

- TP Ferro (France-Spain)

On mid-2017, Vossloh systems have processed more than 1 million measures on monitored turnouts.

Vossloh has carried out several test campaigns and has taken on board the advice, expert assessments and help of SNCF Turnout specialists in order to adjust the powerful detection and defect anticipation algorithms based on representative behavioral indicators characteristic of a family of problems (mechanical, electrical, temporal, etc.).

These indicators have then been analyzed by a trend software package, which checks the relevance of each indicator and performs correlations to determine whether a specific anomaly is indeed a defect or merely a one-off malfunction that it will be necessary to confirm during a subsequent drive.

Vossloh uses its customers' feedback, as well as its own one, to define the best practices for the monitoring of railway assets.

\section{Turnouts monitoring}

\section{Predictive maintenance}

The European standard NF EN 13306 X 60-319 defines the predictive maintenance as "a condition-based maintenance, performed according to the forecasts extrapolated from the analysis and the ratings of the relevant parameters of the asset degradation".

The predictive method aims to reduce the maintenance costs as:

Direct costs: refer to figure 1 comparison of availability times

- One preventive or corrective operation is typically planned and then is shorter than reparation due to corrective maintenance.

- The frequency of the technical operations, using a predictive maintenance, is reduced compared to preventive maintenance. 

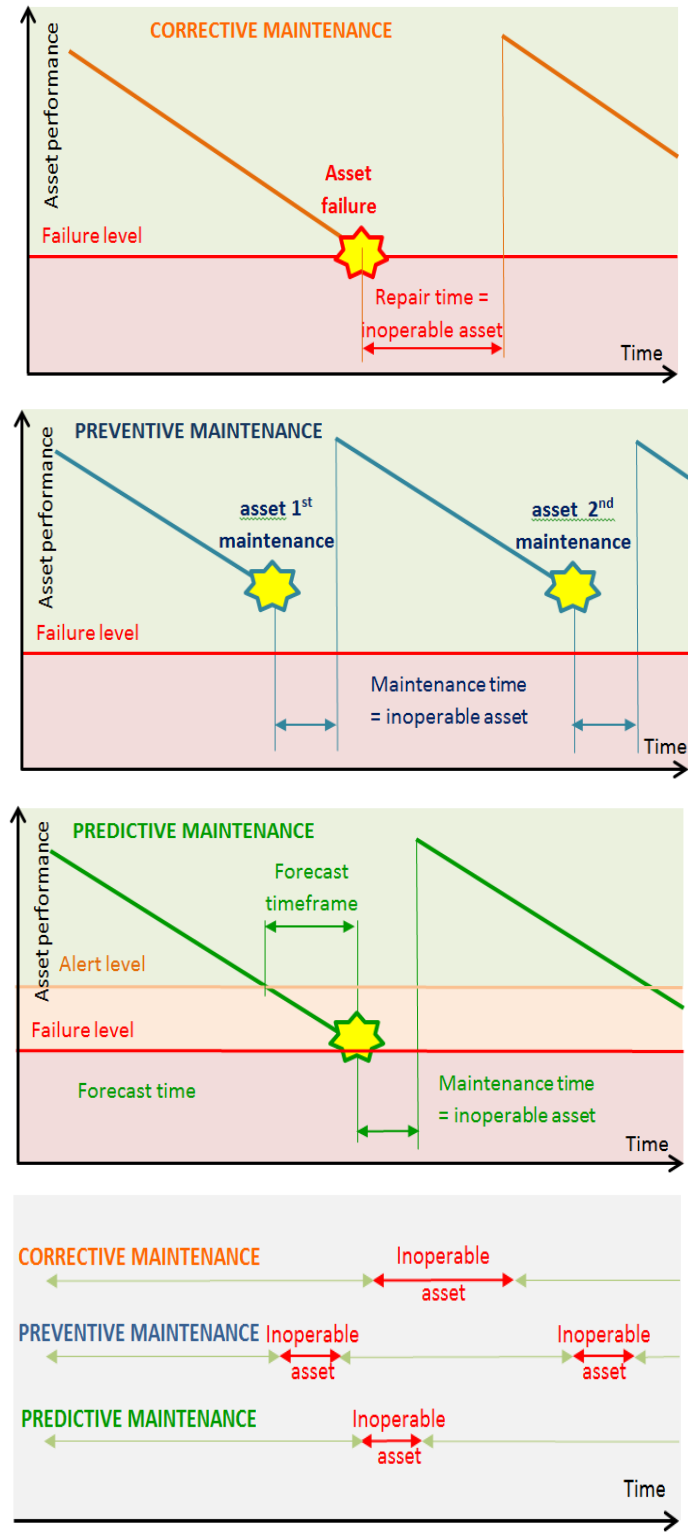

1. Comparison of availability times

Indirect costs:

- The maintenance workload can be planned and smoothed.

- The spare components quantities as well as the urgent orders can be reduced.

- The cost due to the asset unavailability is also optimized.

\section{Why do the turnouts should be monitored?}

Using the predictive maintenance method for the turnouts requires numerous measures. Remote monitoring allows to record them in real-time, without any impact on the train traffic.

The remote monitoring of systems improves train regularity on busy lines:

- Reduction of the failure reporting time,

- Reduction of the routing time through remote consultation of failure types and their principal causes,

- Reduction of work times through facilitated understanding and immediate identification of the action to be taken and the localization.

- Predictive remote monitoring allows improving: 
- Reliability

- Availability

- Maintainability

- Safety

- Maintenance Cost

Based on feedback regarding turnouts, Vossloh has removed several characteristic defects common to several networks. The main ones that can be detected by remote monitoring are:

- Inspection detector:

These faults are inspection system faults, which are mainly due to driving rod problems (track gauge, expansion, loss of adjustment, etc.)

- Lock adjustment:

The turnout is equipped with a clamp/lock type locking system. Adjustment faults are observed.

- Friction:

These defects are detected in the mechanical drive torque limitation element, which is stressed in the event of obstruction, heeling, excessive load, etc. Its loss of adjustment causes either a deterioration of its function (non-protection if too tight) or a limitation of the drive effort capacity.

- Obstacles

Obstacles can become lodged in the point rail or in the mechanical system of the turnout.

- Driving rods/guidance:

The adjustment of the guidance system by driving rods is important, in particular for turnouts without greasing. Losses of adjustment can cause hard points and poor load distribution.

\section{A whole range of measurements}

Vossloh experience leads to use various types of measurements:

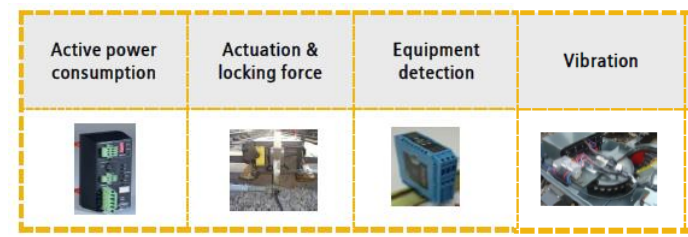

2. Examples of used sensors

- Active power consumption

The measurement of the active power during the switch movement gives correct data regarding turnout behaviour. It is comparable with the driving force which is an indicator usually used by the maintenance team to check correct turnout behaviour.

Power measurement works for both DC and AC point machines. This sensor is the most used by permanent way managers because its location is in the Signaling Equipment Room instead of next to the track.

- Current in inspection circuits 
Measurement of the current in the detection circuit can provide information of the time of non-control of the detection circuit. The detection circuit is used by the signaling system to validate the position of the turnout. During turnout movement, the current cuts out. When the current is cut for a long period it means the position is not safe: the turnout cannot lock in one position or that a detector is out of order.

Monitoring the non-control time can help the maintenance team to check the current in the detection circuit and to establish whether the problem is the detector or is in the turnout (obstruction, for example). The SNCF uses relays in its detection circuit and when the train passes through the turnout it creates micro-failures in the circuit, which decrease the service life of the relays. Monitoring the micro-failures helps the maintenance team to change the relays before they are out of order.

- Actuation and locking force

This measurement is the reference for the maintenance team to state about the correct behavior of the turnout. In all networks across Europe, the maintenance team sets a threshold of force during the switch movement and also for the retaining of the switch blade on the stock rail.

A dynamometric pin is commonly used to measure the force. It replaces the pin between the driving rod and the point machine. This sensor is a safety element because, if it breaks, movement is impossible.

This sensor also detects whether the driving rod has loss of adjustment (overload).

- Vibration for drive mechanism

This measurement gives an indicator of the level of vibration of the point machine during train traffic. This is directly linked to the level of tamping of the turnout. If the level of vibration is too high it can break the component inside the point machine. In many cases, the maintenance team does not have the tools to validate tamping when it has been done. The tamping machine does not come when it is required, but when it is scheduled.

- Impact in turnout crossing

Impact measurement on the crossing allows to provide information about the condition of the check gauge (distance between the checked rail and the common crossing). This safety value is usually measured on site several times a year and the checked rail is a component which needs to be replaced. The replacement of the checked rail directly impacts the train traffic. In some infrastructures it is replaced every year.

Using an accelerometer permits impacts to be recorded without slowing the train traffic.

- Temperature and humidity

The measurement of the rail temperature helps the maintenance team to know the length of blade variation. In some networks, high temperature means that turnout components must be adjusted (locking devices, for example).

The measurement of air temperature and humidity is important for the adjustment of signal processing.

\section{Monitoring system for railways turnouts}

Features:

- A monitoring system for a condition-based maintenance requires at least:

- Measuring the relevant parameters to be monitored (power, tension, vibrations...)

- Relevant thresholds which match the degradations statuses

- Furthermore, additional features must be included for the predictive function: 
- Indicators that rate the parameters. The indicators aim to ease understanding and allow comparisons and operations between various measures of different types.

- Database(s). Most of the time, predictive systems require learning period and longterms analysis. They could thus be considered as "big data" systems. The databases are also mandatory to achieve continuous improvements and feedbacks.

- Predictive algorithms.

Sensors:

Sensors in signaling equipment rooms are easier to set up, nevertheless some sensors have to fitted the turnout, and must be placed at the nearest of the track.

As far as possible, they must produce signals free from outside influence. That's why sensors with 4-20mA output are preferred in most cases. They are standard to be easily supplied and replaced, and they allow the detection of sensor failures. Next generations would be wireless, for both data transmission and power supply, to help maintenance operation on turnouts.

Interface:

Monitoring of the railway assets requires a large amount of measurements and sensors. That's why the system interface has to be synthetic and user friendly to allow maintenance department saving time and focusing on critical assets.

The interface should also be accessible from a standard browser. This means the turnouts can be monitors and checked from outside the server room.

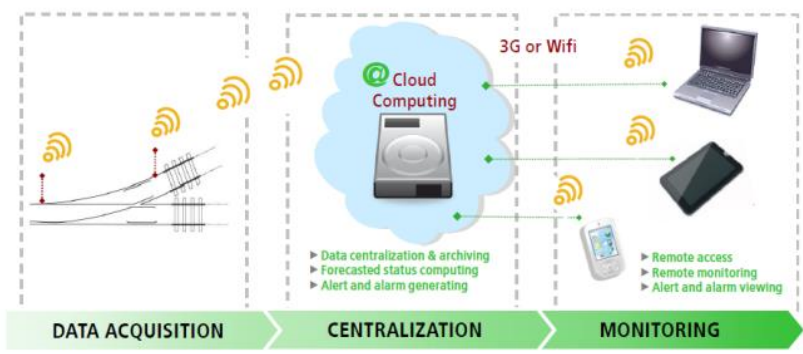

3. Remote interface
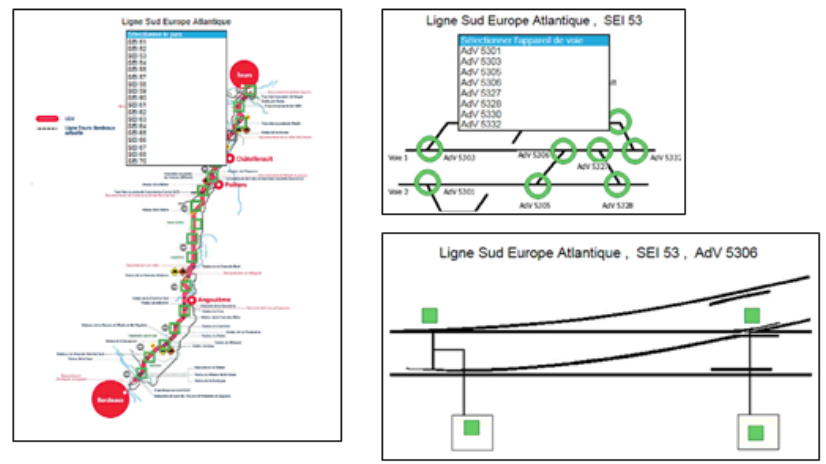

4 User-friendly interface

Architecture:

Data transmission from the measurements points to the server can be achieved according to the site network, by Ethernet, optical fiber, and/or G.SHDSL. 


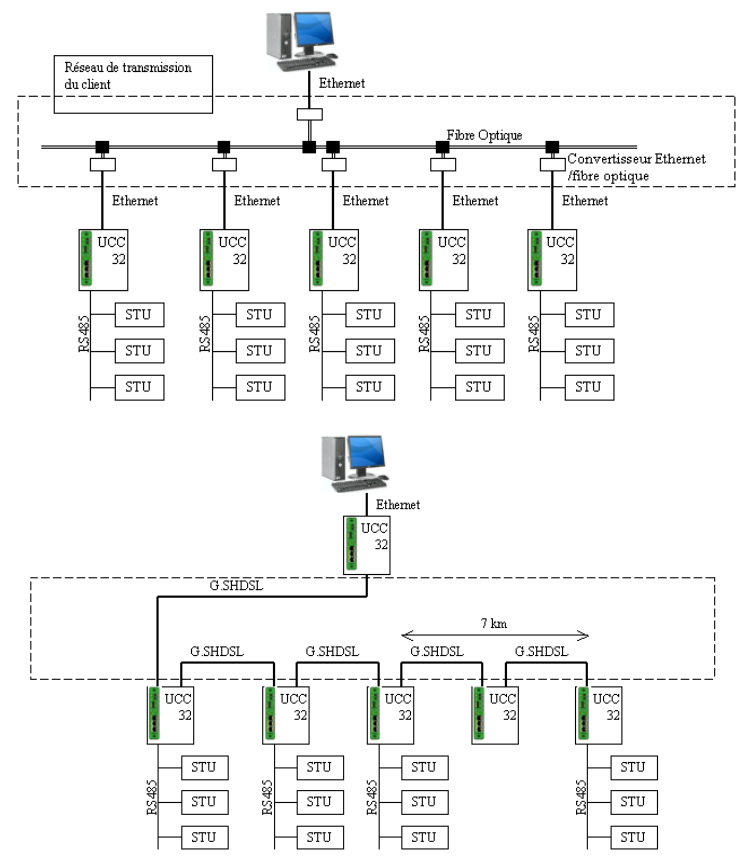

5. Typical architectures

\section{Indicators, alarm \& alert thresholds}

In order to forecast the maintenance operation, the performance of each asset should be graded with at least 3 statuses:

- Operational asset, without any recommendation on maintenance operation.

- Operational asset, with a recommendation on a forecasted maintenance operation.

- An urgent maintenance operation is recommended

The asset can be any monitored equipment: point of measurement, turnout, fleet of turnout. Measures of different types must be reduced to a common performance indicator. It allows to simply define the status, whatever the type of measure. Above all, mathematical operations for the predictive algorithms require a common unit, especially when a turnout uses different types of measures.

- Indicator > 70\%: the asset is operational, no maintenance operation is recommended, status is "ok".

- $70 \%>$ indicator $>30 \%$ : the asset is operational, planning a maintenance operation is recommended. Status is "alert".

- $30 \%$ > indicator: a maintenance operation is strongly recommended as soon as possible. Status is "alarm".

The Vossloh monitoring system typically compares the maximum measured value with the thresholds to define the performance level and indicator.

Figure 6 is an example for the calculation of the indicators from the measured value. Each kind of measure (force, current, power...) has its own thresholds.

Figure 7 shows a measured curve and the associated thresholds. The status depends on the area where the maximum value (in the analyzed time slot) is measured. 


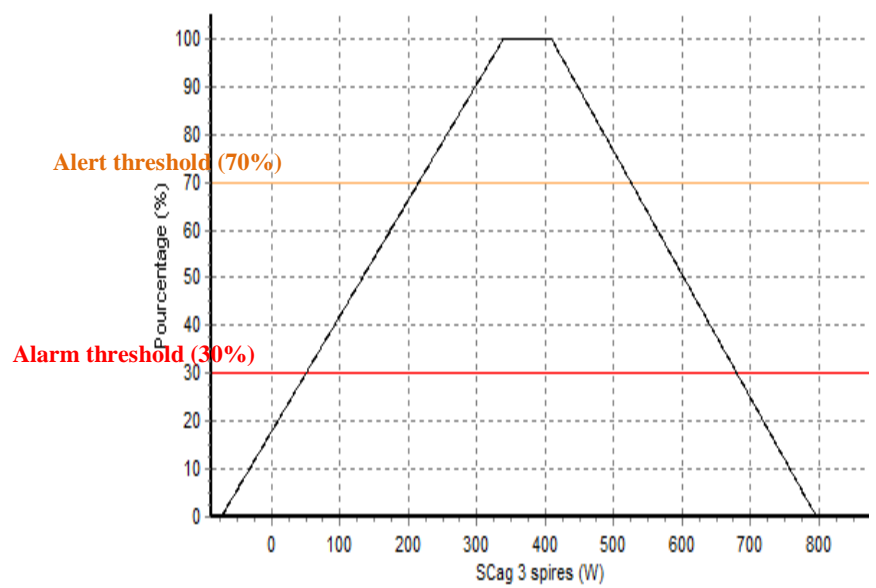

6. Indicators definition

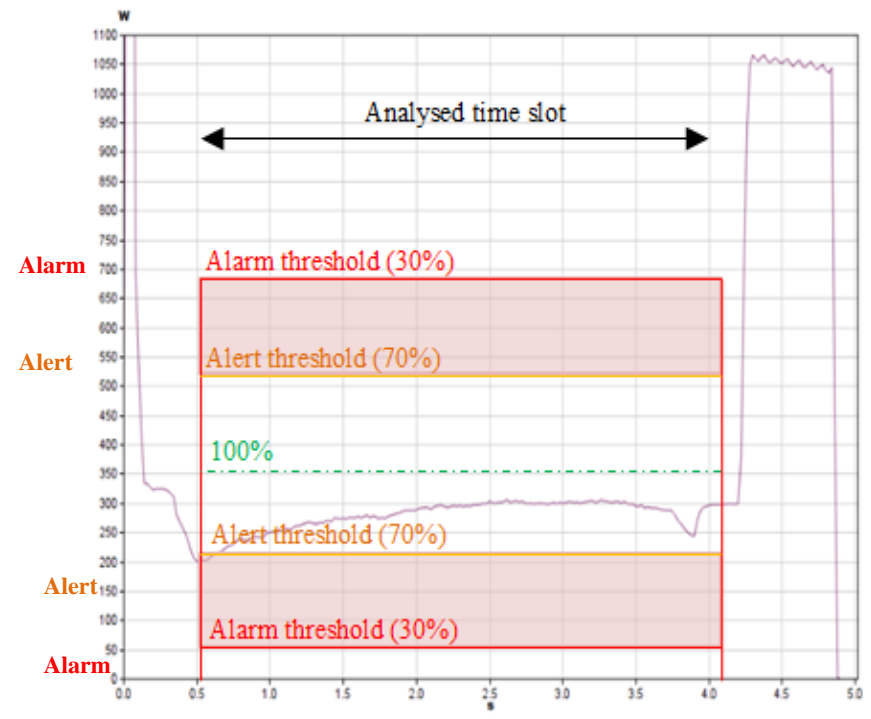

7. Status are defined by indicators

\section{Turnouts predictive analyses}

Each measure can then be graded according to indicators and status. Monitoring the progress of a turnout performance is only possible using comparable measures, that's why one indicator is used for:

- Each turnout

- Each way and direction (to the right / left switching)

- Each phase, typically unlocking / switching / locking for the turnouts with VCC (clamp lock)

A turnout may thus use several indicators.

Mathematical operations are used to calculate an overall turnout indicator and status, from these measures' indicators. These operations are named "rules". Various rules, as minimum, average are applied according to the customer need.

Figure 8 shows the superposition of comparable measures for one month, while figure 9 is a synthetic view of the turnout calculators as a result. 

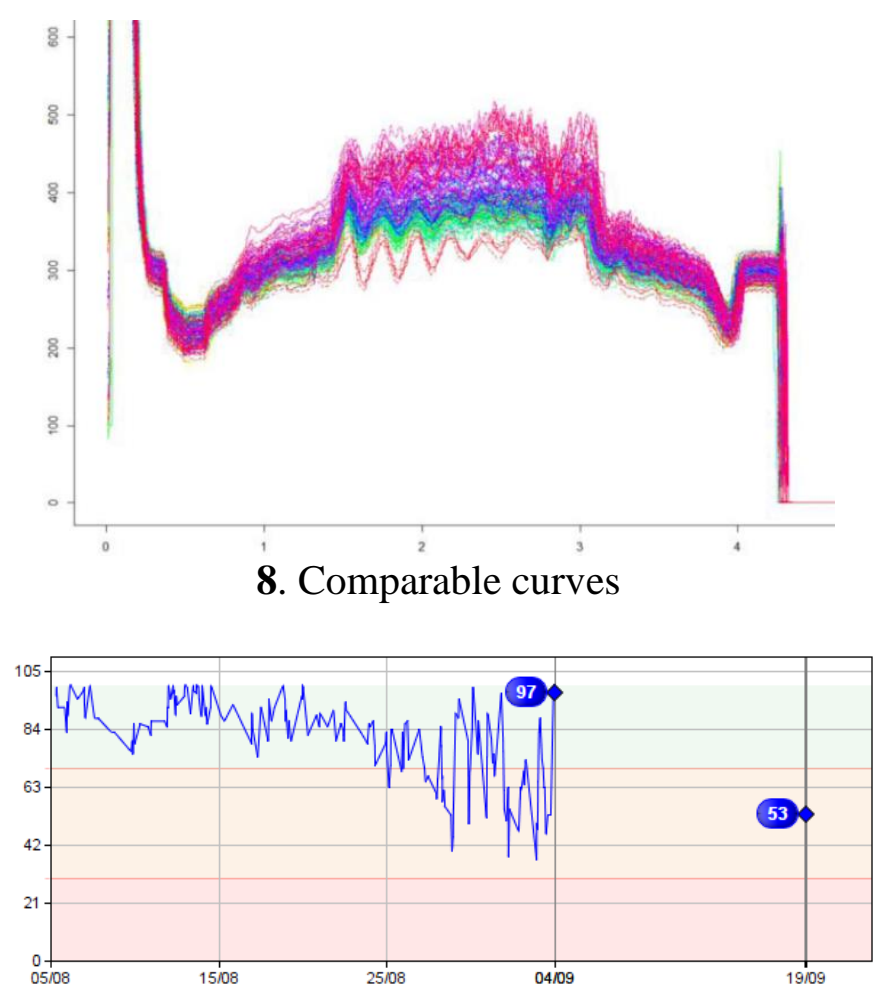

9. Synthetic progress of the turnout indicator

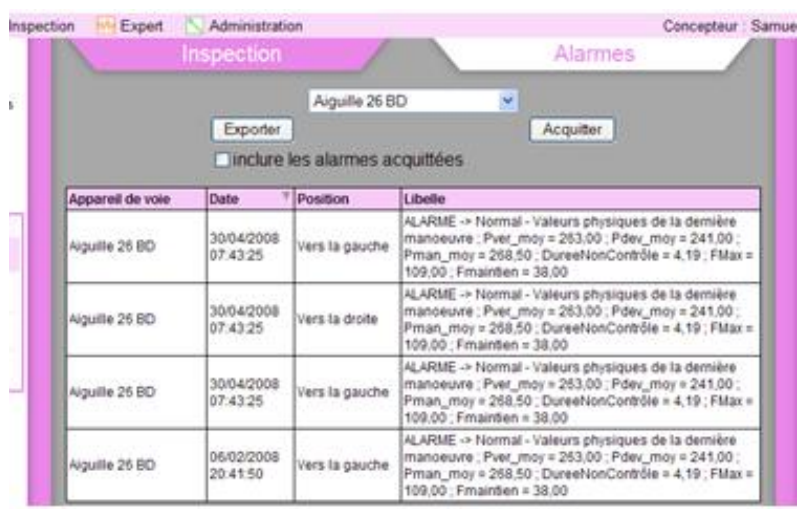

10. Alarm listing

\section{Data mining}

We performed pattern recognition analysis on a complete dataset of measurements from active power consumption of several turnouts. The study aimed at splitting the dataset of comparable measures (same phase / direction / turnout) into 3 classes. Each class represented a status (ok / alert / alarm) and included similar measures. Classification is one of the main tools for automatic learning.

Various methods of classification were used, the two ones described in this document are based on the analysis of the discrete wavelet transform (DWT).

\section{Dataset}

More than 90000 active power measures made up the data set. They were acquired from 32 turnouts, during 15 months. Turnouts switches complied with a normal distribution.

A pretreatment was performed to exclude a few unusual measures from the classification process. Discarded measures fitted with incomplete or irregular switch movements and did not require any predictive algorithms. 


\section{Ascending Hierarchical Classification (AHC)}

The Ascending Hierarchical Classification consists of carrying out progressive grouping of individual values in accordance with their degrees of similarity to obtain a single class that groups them all. Once this calculation has been made, the individual values are divided up into various classes.

A multi-dimensional scaling (MDS) represents the classes in a clearer way. It pointed out that each class is separated from the other ones with obvious boundaries.
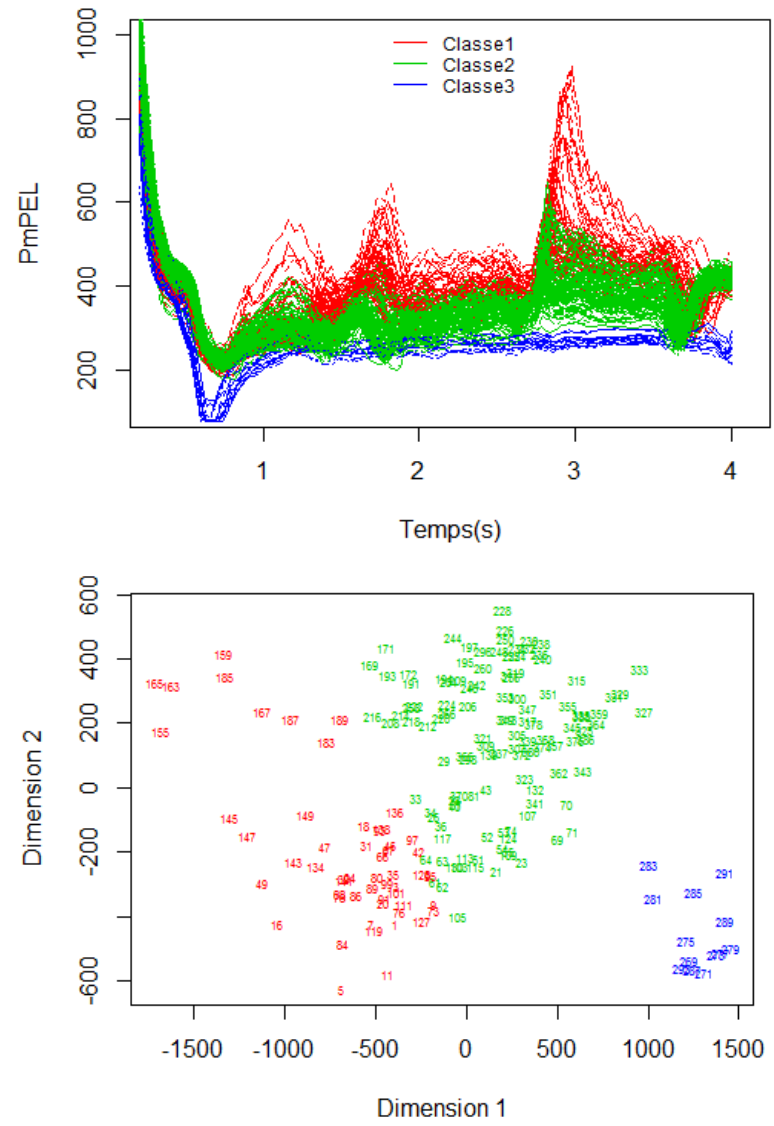

11. AHC classification typical case

\section{Partition Around Medoids (PAM)}

The PAM algorithm partitions the dataset of $\mathrm{n}$ objects into $\mathrm{k}$ clusters. This algorithm works with a matrix of dissimilarity, whose goal is to minimize the overall dissimilarity between the representants of each cluster and its members.

This algorithm is intended to find a sequence of objects called medoids that are centrally located in clusters. In other words, a medoid can be defined as the object of a cluster whose average dissimilarity to all the objects in the cluster is minimal. 

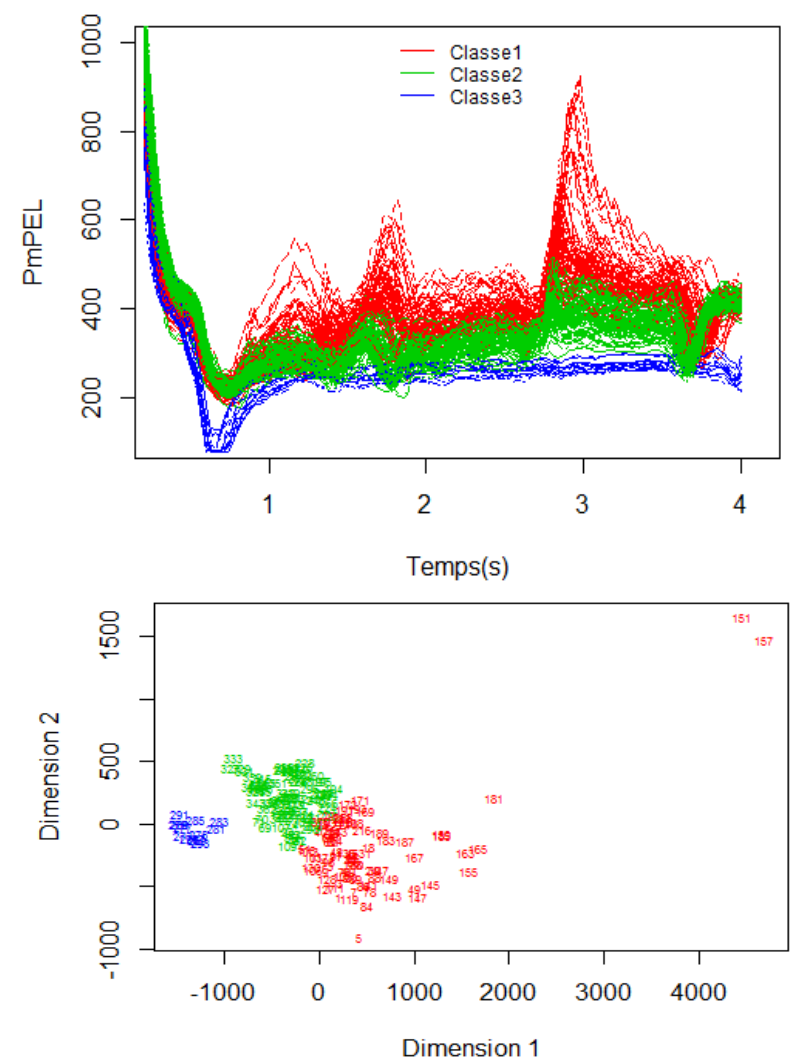

12. PAM classification typical case

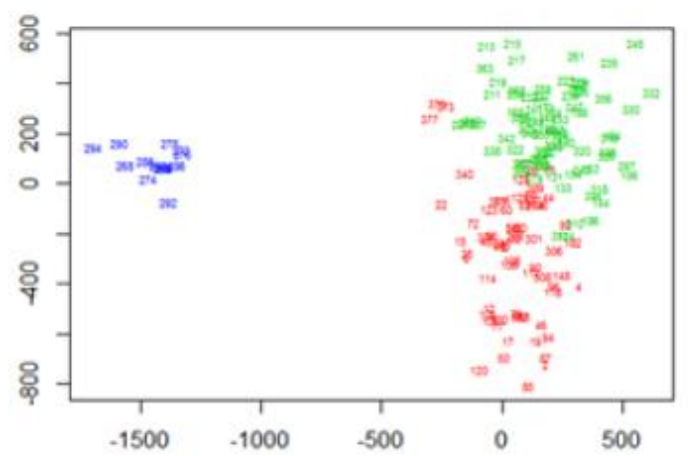

13. Classification irregularities

\section{Results}

Both algorithms, AHC and PAM, gave similar results: classes distributions were comparable, most of the measures could be split into 3 classes. Some classification irregularities occurred (figure 13): a few objects were located outside of their class boundaries. Then some measures could be classified from a class to another one.

Figure 14 shows the probability of changing class, for one of the dataset with the highest quantity of classification anomalies. For instance, $17.4 \%$ of the objects of class 1 might be classified on class $2,82.6 \%$ would remain on class 1 , and there was no probability that objects would be classified class 3 . 


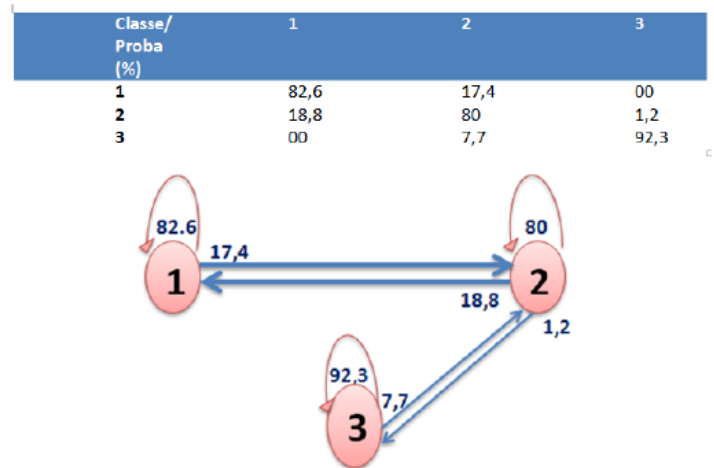

14. Inter-class relations (Markov chains)

\section{Conclusion}

Predictive maintenance costs are often expected to be reduced by $15 \%$. Many points are critical to achieve such a cost reduction:

- A deep technical knowledge about turnouts and their failures modes is necessary to define the system, in particular to define the required type of measures and sensors. Sharing this knowledge and a full cooperation between the maintenance contractor and the monitoring system provider will lead to successful project and effective system.

- The predictive method is more effective with large measurement datasets: algorithms require learning period, while forecasting the usual maintenance operations needs continuous and regular measures.

- A friendly user and synthetic interface are the keys to focus only on critical turnouts. The interoperability with other systems also matters to transmit the alarm to the right person.

- Automatic learning can be achieved though data mining and classification.

- Data mining would also allow to improve preventive algorithms, notably by studying the speeds of the degradations and performances loss. A Markov approach is one of the main tracks to achieve it. 